\title{
The radio surroundings of the microquasar GRO J1655-40
}

\author{
J. A. Combi ${ }^{1}$, G. E. Romero ${ }^{1}$, P. Benaglia ${ }^{1}$, and I. F. Mirabel ${ }^{2,3}$ \\ 1 Instituto Argentino de Radioastronomía, C.C.5, (1894) Villa Elisa, Buenos Aires, Argentina \\ 2 CEA/DMS/DAPNIA/Service D'Astrophysique, Centre d'Études de Saclay, 91191 Gif-sur-Yvette, France \\ 3 Instituto de Astronomía y Física del Espacio (IAFE), C.C. 67, Suc. 28, Buenos Aires, Argentina
}

Received 12 February 2001 / Accepted 22 February 2001

\begin{abstract}
We report the results of a study of the radio surroundings of the superluminal microquasar GRO J1655-40. We have searched for extended continuum structures that might be indicative of the presence of a supernova remnant (SNR) associated with the formation of the compact object in the binary system. We also carried out HI-line observations of the region looking for a local minimum created by an explosive event. Our results indicate that there is, in fact, a bubble in the large-scale HI distribution around GRO J1655-40. We suggest that this structure might be created by the original supernova explosion occurred a few hundred thousand years ago and whose signatures can be traced by the overabundance of $\alpha$-elements recently found by Israelian et al. (1999) in the companion star.
\end{abstract}

Key words. X-rays: stars - stars: evolution - ISM: bubbles - ISM: supernova remnants

\section{Introduction}

The soft X-ray transient GRO J1655-40 is one of the best black hole candidates in the Galaxy (Mirabel \& Rodriguez 1999). It is a low mass X-ray binary whose compact object has a mass in the range 5.5-7.9 $M_{\odot}$ (Shahbaz et al. 1999). The system is located at $3.2 \mathrm{kpc}$ from the Earth and presents frequent superluminal ejections at radio wavelengths (e.g. Tingay et al. 1995; Hjellming \& Rupen 1995).

Recently, Israelian et al. (1999) found convincing evidence of a supernova origin for the black hole in GRO J1655-40. They detected a clear overabundance of $\alpha$-elements in the secondary star, whose internal temperatures are not high enough as to synthesize them. These elements seem to have been produced in a massive companion (with $25-40 M_{\odot}$ ) which exploded both contaminating the secondary star with matter enriched with nucleosynthetic products and creating the black hole. Israelian et al. (1999) estimated that the explosion should have occurred less than $10^{6}$ years ago.

In this Letter, we present the results of a study of the radio environment of GRO J1655-40. Our aim is to detect any existent signature of an explosive event in this region. A supernova explosion should have introduced modifications in the ISM around the binary system, displacing material and creating a low density bubble. Additionally, the expanding shock front should accelerate electrons up to relativistic energies, producing a shell-type

Send offprint requests to: J. A. Combi, e-mail: pauletich@sinectis.com.ar source in the radio continuum. If the age of the system is less than $10^{6}$ years, these observational features might be detectable.

\section{Data analysis and new observations}

\subsection{Radio continuum}

We have used data from the $4.85-\mathrm{GHz}$ PMN survey (Condon et al. 1993) in order to produce a large-scale map $\left(\sim 1^{\circ} \times 1^{\circ}\right)$ of the surroundings of GRO J1655-40. Although these data are optimized for sizescales of less than 40 arcmin, the good resolution and sensitivity of the images render them a valuable tool to search for SNR candidates, especially if they are not of large angular size (Duncan et al. 1997). For this work, we apply to the $4.85-\mathrm{GHz}$ data an additional filtering process in order to remove the galactic diffuse emission on scales larger than 30 arcmin (see Combi et al. 1998 for details of the Gaussian filtering method). The resulting map is shown in Fig. 1, along with a 1.4-GHz image of the small-scale emission of the inner region obtained from the NVSS Sky Survey (Condon et al. 1998) at a better resolution. These latter observations were obtained with the Very Large Array (VLA) in the compact D and DnC configurations.

\subsection{HI observations}

We have performed HI observations towards the area of interest with a $30-\mathrm{m}$ single dish telescope located at 

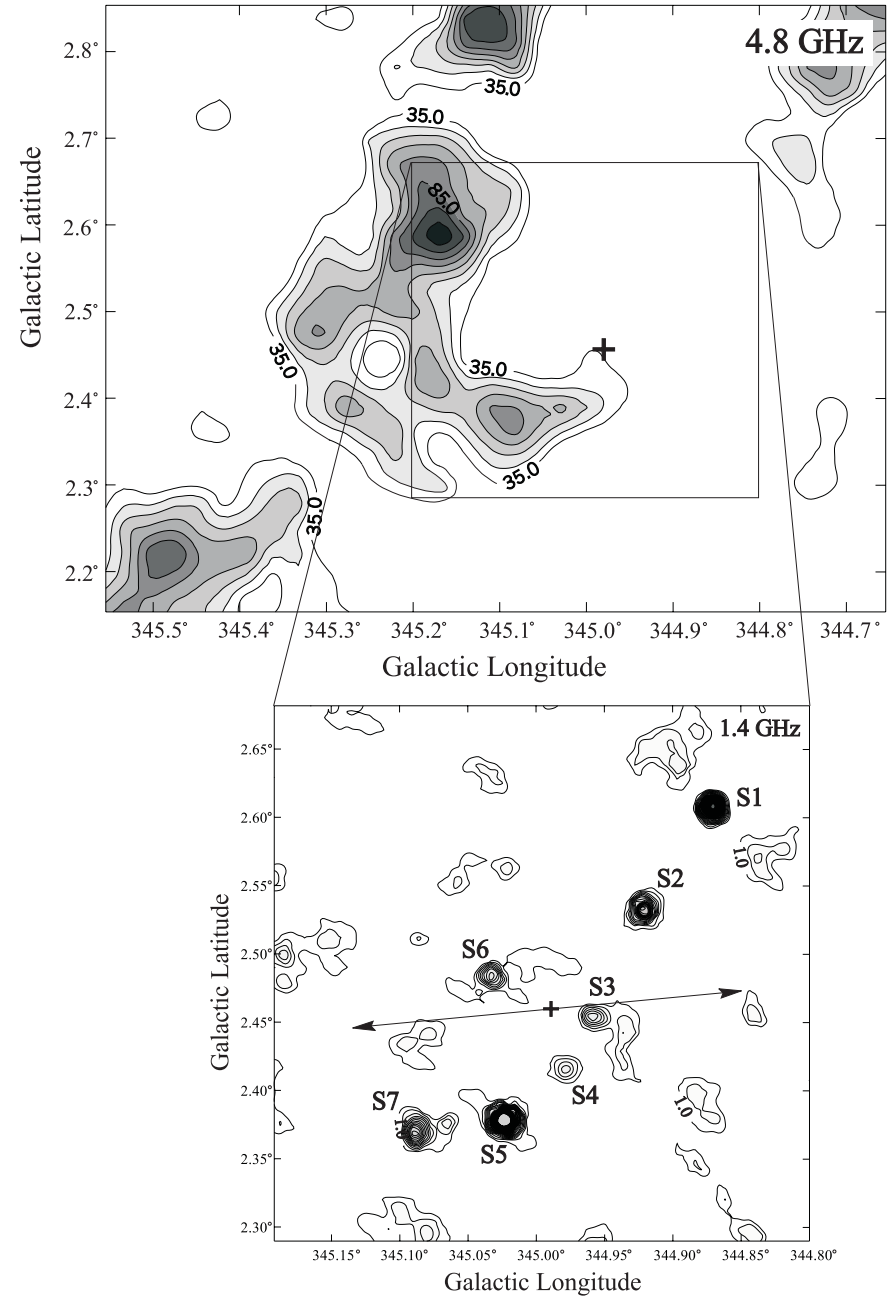

Fig. 1. Upper panel: filtered radio emission at $4.85 \mathrm{GHz}$ of the region surrounding GRO J1655-40. The position of the microquasar is marked by a cross. Contours are labeled in steps of $10 \mathrm{mJy}$ beam $^{-1}$, starting from $35 \mathrm{mJy}$ beam $^{-1}$. Lower panel: small-scale, higher resolution, VLA image at $1.4 \mathrm{GHz}$ for the region close to GRO J1655-40. Radio contours are in steps of $2 \mathrm{mJy}_{\text {beam }}{ }^{-1}$ starting from $1 \mathrm{mJy} \mathrm{beam}^{-1}$. The arrows indicate the directions of the observed superluminal ejections

the Instituto Argentino de Radioastronomía (IAR), Villa Elisa, Argentina. The observations were carried out during four consecutive sessions on November 20-23, 2000. The receiver is a helium-cooled HEMT amplifier with a 1008channel autocorrelator at the backend. System parameters and additional details of the observational technique can be found in Combi et al. (1998). The HI line was observed in hybrid total power mode and the sky was sampled on a $0.35^{\circ}$ rectangular grid. Each grid position was observed during $60 \mathrm{~s}$ with a velocity resolution of $\sim 1 \mathrm{~km} \mathrm{~s}^{-1}$ and a coverage of $\pm 450 \mathrm{~km} \mathrm{~s}^{-1}$. A set of HI brightness temperature maps $\left(\Delta T_{\mathrm{rms}} \sim 0.2 \mathrm{~K}\right)$ were made for the velocity interval $\Delta v=\left(-80 \mathrm{~km} \mathrm{~s}^{-1},+20 \mathrm{~km} \mathrm{~s}^{-1}\right)$. Those maps for the interval -42 to $-30 \mathrm{~km} \mathrm{~s}^{-1}$, corresponding to the HI distribution at a distance centered at $\sim 3.5 \mathrm{kpc}$, are shown in Fig. 2.
Table 1. Point radio sources near GRO J1655-40

\begin{tabular}{lccc}
\hline Source & $\begin{array}{c}(l, b) \\
(\mathrm{deg}, \mathrm{deg})\end{array}$ & $\begin{array}{c}F_{1.4 \mathrm{GHz}} \\
(\mathrm{mJy})\end{array}$ & ID \\
\hline S1 & $\left(344.87^{\circ},+2.62^{\circ}\right)$ & 24.9 & - \\
S2 & $\left(344.92^{\circ},+2.54^{\circ}\right)$ & 12.8 & - \\
S3 & $\left(344.96^{\circ},+2.46^{\circ}\right)$ & 5.43 & - \\
S4 & $\left(344.98^{\circ},+2.42^{\circ}\right)$ & 4.48 & - \\
S5 & $\left(345.02^{\circ},+2.38^{\circ}\right)$ & 37.7 & - \\
S6 & $\left(345.03^{\circ},+2.49^{\circ}\right)$ & 5.83 & - \\
S7 & $\left(345.10^{\circ},+2.37^{\circ}\right)$ & 10.6 & PMN J1654-3949 \\
\hline
\end{tabular}

\section{Main results}

Figure 1 (upper panel) shows the radio continuum image at $4.85 \mathrm{GHz}$ of the surroundings of GRO J1655-40. A small $\left(\sim 24^{\prime} \times 24^{\prime}\right)$ semi-circular radio source, which very much resembles a SNR, can be clearly seen in the center of the image. This extended, partial shell source is centered at $(l, b) \approx\left(345.14^{\circ},+2.48^{\circ}\right)$. It has an integrated flux density of $\sim 0.5 \mathrm{Jy}$ at $4.85 \mathrm{GHz}$, although uncertainties due to the background filtering make difficult to estimate a realistic value. The microquasar is located at a distance of $\sim 0.15 \mathrm{deg}$ from the center of the radio source.

Figure 1 (lower panel) shows the VLA image at $1.4 \mathrm{GHz}$ (Condon et al. 1998) of the vicinity of GRO J1655-40. The microquasar was undetectable at the epoch of the observations and its position is marked with a cross. There are seven point sources near the microquasar with fluxes above $5 \mathrm{mJy}$. They have been labeled from S1 to S7 in the image (notice that the SNR candidate is not visible in this map because these data are optimized for point source detection). The bright, compact source located at $(l, b) \sim 345.1^{\circ},+2.37^{\circ}$ is identified in the PMN catalogue as PMN J1654-3949. The sources S1 to S6 have no entry in any point source catalog at present. The measured characteristics of all these sources are summarized in Table 1.

Figure 2 clearly shows the development of a cavity around GRO J1655-40 in the HI maps from -42 to $-30 \mathrm{~km} \mathrm{~s}^{-1}$. Although the cavity do not form a shell due to the steep density gradient towards the galactic plane, the existence of a local minimum in the HI distribution at the position of the microquasar is evident. This kind of "horse-shoe" morphology has been observed around earlytype stars with very strong stellar winds (e.g. Benaglia \& Cappa 1999), where a strong energy release in a highly inhomogeneous medium has occurred.

In Fig. 3 we present the integrated column density map for the velocity interval from -40 to $-30 \mathrm{~km} \mathrm{~s}^{-1}$. Standard galactic rotation models (Fich et al. 1989) indicate that this HI hole is located at a kinematic distance of 3 to $4 \mathrm{kpc}$, in good accordance with the estimated distance to GRO J1655-40. 

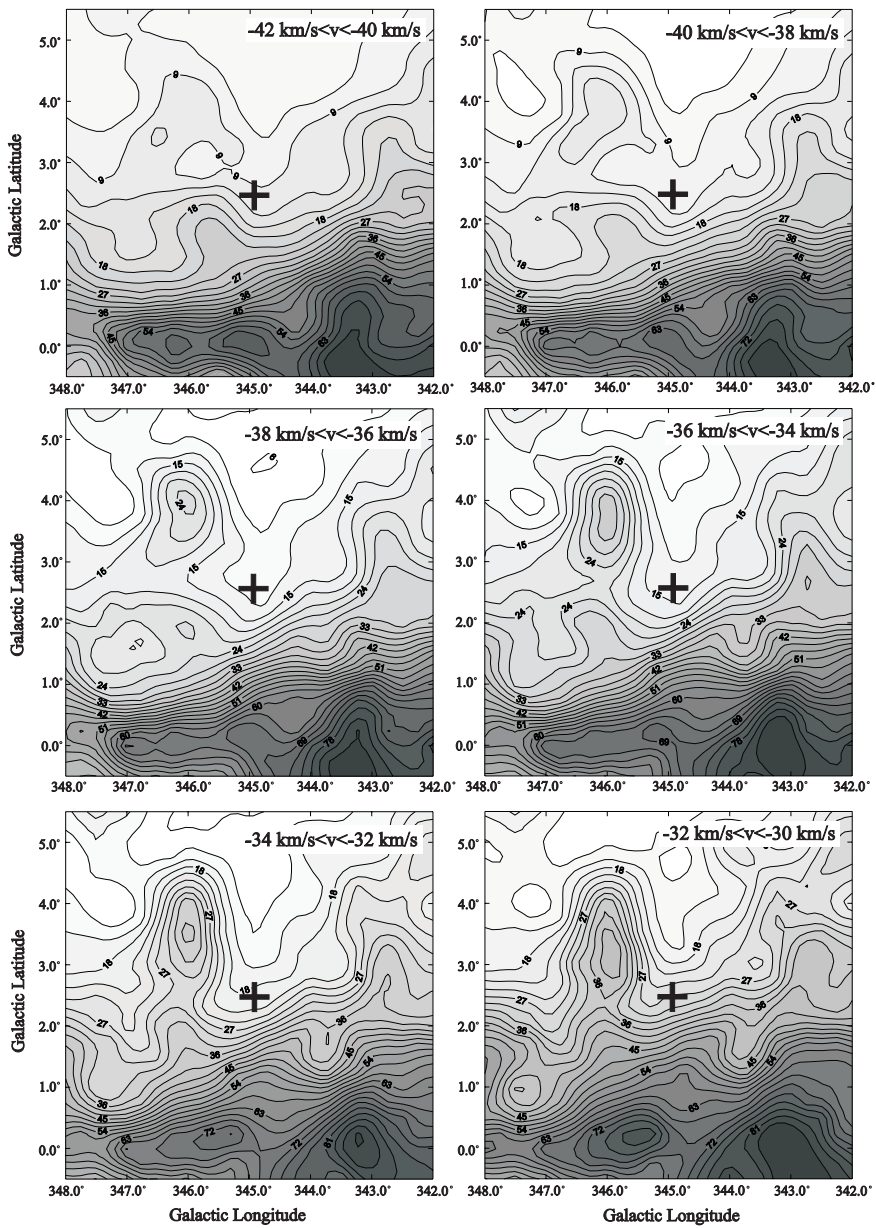

Fig. 2. HI brightness temperature channel maps (contour labels in $\mathrm{K}$ ) obtained for the velocity range from -42 to $-30 \mathrm{~km} \mathrm{~s}^{-1}$ around the position of GRO J1655-40 (indicated with a cross). Due to the differential galactic rotation the expected velocity of the interstellar medium at the distance of $3.2 \mathrm{kpc}$ of the X-ray binary is $\sim 38 \mathrm{~km} \mathrm{~s}^{-1}$

\section{Discussion}

If we assume that the extended radio structure shown in Fig. 1, upper panel, is a supernova remnant located at the same distance than the microquasar, we get that its radius should be $\sim 15 \mathrm{pc}$. The surface brightness of the source results to be abnormally low for such a remnant $\left(\Sigma_{4.8 \mathrm{GHz}} \sim 1.310^{-22} \mathrm{~W} \mathrm{~m}^{-2} \mathrm{~Hz}^{-1} \mathrm{sr}^{-1}\right)$. On the other hand, the microquasar is displaced about $0.15^{\circ}$ from the center of the semi-shell source. Although the transverse velocity of GRO J1655-40 is not known, it is constrained to be less than $300 \mathrm{~km} \mathrm{~s}^{-1}$ (Brandt et al. 1995). At this velocity, it would have taken more than $2.710^{4} \mathrm{yr}$ to reach its current position. And for a velocity similar to the observed radial velocity $\left(\sim 114 \mathrm{~km} \mathrm{~s}^{-1}\right)$, the age of the system would be $\sim 82500$ yr. These timescales are incompatible with the size of the remnant and our knowledge of the ambient density in this region (see below), and lead us, consequently, to discard the extended radio source in Fig. 1 as possibly associated with GRO J1655-40. This

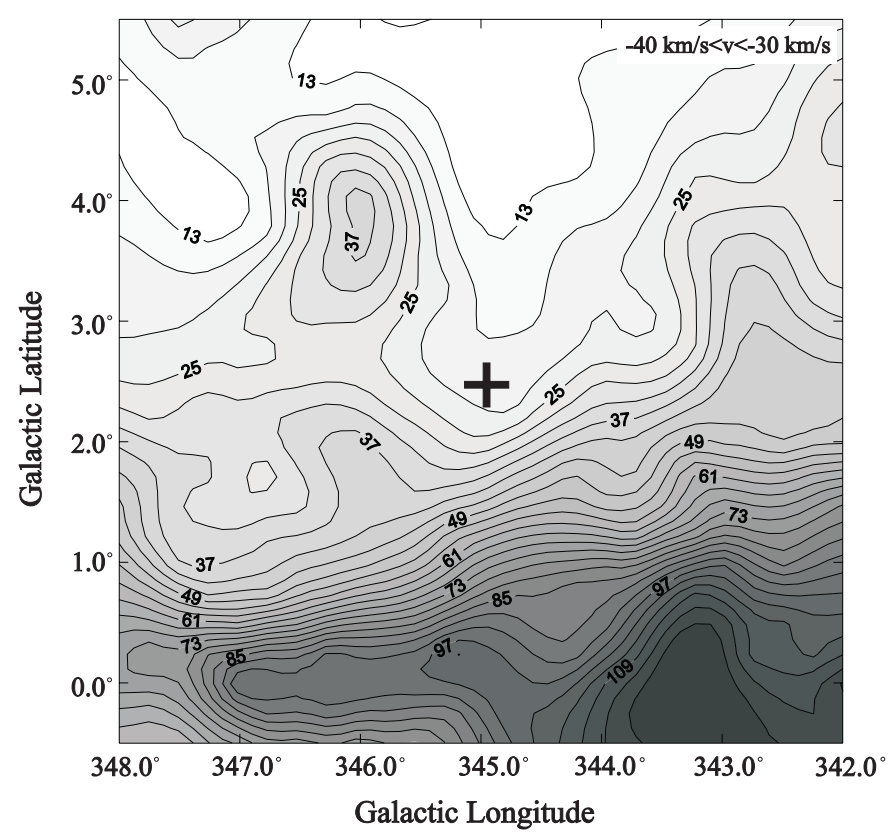

Fig. 3. Integrated column density map for the velocity range from -40 to $-30 \mathrm{~km} \mathrm{~s}^{-1}$. Contour labels are in units of $10^{19} \mathrm{~cm}^{-2}$. Microquasar position indicated with a cross

source could be a background SNR or, perhaps, an artifact formed by several close and unresolved point sources.

At larger sizescales there are not other clear continuum structures that could be associated with a SNR. We have inspected larger maps, of $\sim 6^{\circ} \times 6^{\circ}$, in search of additional signatures, but nothing was found. This is a very confused region located nearly towards the Galactic Center and the detection of weak continuum features with low surface brightness is extremely difficult. Even at small scales there is a significant number of sources, as it is shown in Fig. 1, lower panel. Here, the two closest sources to the microquasar, S3 and S6, seem to be at approximate equal angular distances, in opposite directions, of the X-ray binary. However, it is unlikely that they could be old emission knots ejected by the central object, because they are on an axis displaced $30^{\circ}$ from the axis determined by the known ejections (Hellming \& Rupen 1995), which is also shown in the figure.

Contrary to the often misleading continuum data, the $\mathrm{HI}$ observations can provide more clear signatures of an explosive event in this region, because the differential rotation motion of the material allows a glimpse of the matter distribution along the line of sight. The channel maps in Fig. 2 show the development in the velocity distribution of a cavity in the HI with a "horse-shoe" shape and an average radius of $\sim 75 \mathrm{pc}$ at a distance of $3.2 \mathrm{kpc}$. The integrated column densities shown in Fig. 3 can be used to estimate the mass displaced to form the cavity. Assuming that the background density is given by the density at the minimum, we estimate that at least $25000 M_{\odot}$ have been removed from this region. Since the average expansion velocity of the shell is $\sim 15 \mathrm{~km} \mathrm{~s}^{-1}$, we obtain that a lower limit to the original energy release which started the mass 
motion at the center of the cavity is $\sim 610^{49} \mathrm{erg}$. The expansion velocity should have been considerably higher in the past, in such a way that the real value is probably in the range $10^{50}-10^{51} \mathrm{erg}$, what is quite consistent with the supernova hypothesis. This is reinforced by the fact that there are not known early-type stars with stellar winds strong enough to create such a bubble in this sector of the sky. It is noteworthy that even higher values for the initial energy release are possible if the explosion was caused by a hypernovae event as recently proposed by Brown et al. (2000).

The mean number density in this region can be obtained averaging the column densities within the bubble. This provides a rough estimate of the average density of the ISM before the explosion. We obtain a value $n_{0} \sim 0.4 \mathrm{~cm}^{-3}$. This density and the size of the cavity ( $R \sim 75 \mathrm{pc}$ ) indicate that the SNR should be well within the radiative phase. An exact estimate of the time elapsed since the explosion is not possible without a knowledge of the detailed structure of the ISM in this region, but simulations with an original energy release of few times $10^{50} \mathrm{erg}$ and some reasonable conditions for the cloud properties suggest ages in the range $10^{5}-10^{5.5} \mathrm{yr}$ (Cowie et al. 1981). The continuum emission of the remnant, with sizescales of several degrees, should have been removed during the filtering of the background radiation in the PMN survey. New observations with good sensitivity and single dish telescopes over a large area are necessary to detect the SNR radio structure and help to make a more accurate estimate of its age, if the remnant is not already dissipated.

In summary, we have presented radio continuum and HI maps of the surroundings of the black hole candidate GRO J1655-40. A bubble centered at the microquasar has been detected in our HI data. The energy necessary to create this bubble and its approximate age are consistent with the hypothesis that it is the trace left in the ISM by the supernova explosion occurred when the black hole was formed.

Acknowledgements. This work was supported by CONICET (PIP 0430/98), ANPCT (PICT 03-04881) and Fundación Antorchas.

\section{References}

Benaglia, P., \& Cappa, C. E. 1999, A\&A, 346, 979

Brandt, W. N., Podsiadlowski, Ph., \& Sigurdsson, S. 1995, MNRAS, 277, L35

Brown, G. E., Lee, C.-H., Wijers, R. A. M. J., et al. 2000, New Astron., 5, 191

Combi, J. A., Romero, G. E., \& Benaglia, P. 1998, A\&A, 333, L91

Condon, J. J., Griffith, M. R., \& Wright, A. E. 1993, AJ, 106, 1095

Condon, J. J., Cotton, W. D., Greisen, E. W., et al. 1998, AJ, 115,1693

Cowie, L. L., McKee, C. F., \& Ostriker, J. P. 1981, ApJ, 247, 908

Duncan, A. R., Stewart, R. T., Haynes, R. F., \& Jones, K. L. 1997, MNRAS, 287, 722

Fich, M., Blitz, L., \& Stark, A. A. 1989, ApJ, 342, 272

Hjellming, R. M., \& Rupen, M. P. 1995, Nature, 375, 464

Israelian, G., Rebolo, R., Basti, G., Casares, J., \& Martin, E. L. 1999, Nature, 401, 142

Mirabel, I. F., \& Rodríguez, L. F. 1999, ARA\&A, 37, 409

Shahbaz, T., van der Hooft, F., Casares, J., Charles, P. A., \& van Paradijs, J. 1999, MNRAS, 306, 89

Tingay, S. J., et al. 1995, Nature, 374, 141 